\title{
Impulsive Excitation of Phonon-Pair Combination States by Second-Order Raman Scattering
}

\author{
Albrecht Bartels,* Thomas Dekorsy, and Heinrich Kurz \\ Institut für Halbleitertechnik, Rheinisch-Westfälische Technische Hochschule Aachen, \\ Sommerfeldstrasse 24, D-52056 Aachen, Germany
}

\begin{abstract}
The excitation of (100)-oriented $\mathrm{KTaO}_{3}$ with 25-fs laser pulses impulsively drives phonon-pair combination states via second-order Raman scattering. Oscillations in the phonon-amplitude covariance at the sum and difference frequency of the two involved phonons are observed in a spectrally and temporally resolved pump-probe experiment. Transmission changes of the sample are dominated by contributions of wave vector conserving phonon-pair combinations from the entire Brillouin zone that have maxima in their combined density of states. For low temperatures the temperature dependence of the covariance oscillations of different phonon combinations is reproduced by a quantum-mechanical model.
\end{abstract}

The investigation of coherent phonons in solids has been a field of extensive research since the first observation of coherent phonons in semiconductors [1]. Several excitation mechanisms for coherent lattice vibrations in solids have been identified until now [2]. In the framework of first-order impulsive stimulated Raman scattering coherent phonons are characterized by a nonzero expectation value of the phonon-amplitude oscillating with their eigenfrequency. The driving laser pulse reduces the uncertainty in the amplitudes and the canonical momenta. However, coherent states do not exhibit dynamics in statistic variables like amplitude variances and covariances of phonon modes. Different eigenmodes of the lattice remain uncorrelated.

A route to induce macroscopically detectable dynamics in the statistical properties of the lattice is second-order Raman scattering (SOR). Recently, vacuum squeezing of phonons generated by SOR has been predicted by $\mathrm{Hu}$ and Nori [3] and demonstrated experimentally by Garret et al. in $\mathrm{KTaO}_{3}$ [4]. They found that the uncertainties of the phonon amplitude and canonical momentum oscillate out of phase at twice the fundamental phonon frequency and temporarily drop below their vacuum noise level. $\mathrm{KTaO}_{3}$ is ideally suited for studies on SOR, since for symmetry reasons it exhibits only second-order but no first-order Raman scattering. Thus the excitation of dynamics in the statistical properties of a solid such as amplitude variances (in general associated with squeezed states) and covariances of phonon-pair amplitudes (in general associated with correlations of phonon pairs) can be investigated directly. In this Letter we report on the observation of such combination states of phonon pairs generated by ultrashort light pulses via SOR in a solid: Two oscillators that are decoupled in the absence of perturbation (neglecting anharmonicities) become correlated.

To model the second-order Raman excitation in $\mathrm{KTaO}_{3}$ we consider two harmonic oscillators with amplitudes $Q_{1}, Q_{2}$ and canonical momenta $P_{1}, P_{2}$ coupled via the second-order polarizability $\alpha^{i j}=\partial^{2} \alpha / \partial Q_{i} \partial Q_{j}$ by excitation with a linearly polarized optical pulse with electric field component $E(t)$. The unperturbed Hamiltonian $H_{0}=$
$\left(P_{1}^{2}+\omega_{1}^{2} Q_{1}^{2}+P_{2}^{2}+\omega_{2}^{2} Q_{2}^{2}\right) / 2$ and the perturbation contribution $H_{s}=-\frac{1}{4}|E(t)|^{2} \sum_{i, j=1}^{2} \alpha^{i j} Q_{i} Q_{j}$ combine to the complete Hamiltonian $H=H_{0}+H_{s}$. $\quad \alpha^{i j}$ reflects the second-order term in the expansion of the electronic susceptibility in powers of atomic displacements.

Before the pulse strikes, both oscillators are assumed to be in eigenstates of the unperturbed Hamiltonian $\left|n_{1}\right\rangle$ and $\left|n_{2}\right\rangle$ where $n_{1}$ and $n_{2}$ are the occupation numbers of oscillators 1 and 2, respectively. The combined wave function is written $\left|\psi^{-}\right\rangle=\left|n_{1}, n_{2}\right\rangle$. Since our experimental pulse length is much shorter than the periods of the phonon modes involved and the time it takes the pulse to pass the sample, a Dirac-like excitation $|E|^{2}=4 \pi I_{0} \delta(t) / n c$ can be assumed for simplicity, where $I_{0}$ is the integrated intensity of the pulse, $n$ is the refractive index of the material, and $c$ is the speed of light. Introducing $\xi^{i i}=$ $-\pi I_{0} \alpha^{i i} / n c \omega_{i} \quad($ with $\quad i=1,2) \quad$ and $\quad \xi^{12}=\xi^{21}=$ $-2 \pi I_{0} \alpha^{12} / n c \sqrt{\omega_{1} \omega_{2}}$, the perturbation Hamiltonian can be defined by $H_{s}=V_{s} \delta(t)$ with $V_{s}=\left[\xi^{11} \omega_{1} Q_{1}^{2}+\right.$ $\left.\xi^{22} \omega_{2} Q_{2}^{2}+\xi^{12} \sqrt{\omega_{1} \omega_{2}} Q_{1} Q_{2}\right]$. This allows one to expand the wave function after interaction with the pulse $\left|\psi^{+}\right\rangle$into a series:

$$
\left|\psi^{+}\right\rangle=\left(\sum_{n=0}^{\infty} \frac{1}{(i \hbar)^{n}} V_{s}^{n}\right)\left|\psi^{-}\right\rangle .
$$

Under our experimental conditions, the $\xi^{i j}$ are in the order of $10^{-4}$ only. The truncation of $\left|\psi^{+}\right\rangle$after terms of first order in the $\xi^{i j}$ leads finally to the wave function after excitation:

$$
\begin{aligned}
\left|\psi^{+}\right\rangle= & \left\{1-\frac{i}{2}\left[\xi^{11}\left(a_{1}+a_{1}^{\dagger}\right)^{2}+\xi^{22}\left(a_{2}+a_{2}^{\dagger}\right)^{2}\right.\right. \\
& \left.\left.+\xi^{12}\left(a_{1}+a_{1}^{\dagger}\right)\left(a_{2}+a_{2}^{\dagger}\right)\right]\right\}\left|\psi^{-}\right\rangle .
\end{aligned}
$$

Here, the oscillator amplitudes and canonical momenta in $V_{s}$ were replaced by the respective phonon creation and annihilation operators $a_{i}^{\dagger}$ and $a_{i}$. This wave function now contains only perturbation contributions resulting from the simultaneous interaction of the light pulse with two phonons. The amplitude expectation values vanish $\left(\left\langle\psi^{+}\left|Q_{i}\right| \psi^{+}\right\rangle=\left\langle Q_{i}\right\rangle=0\right)$; i.e., no coherent phonons are 
generated. The variances $\left\langle\left(Q_{i}-\left\langle Q_{i}\right\rangle\right)^{2}\right\rangle=\left\langle Q_{i}^{2}\right\rangle$ and the covariance $\left\langle\left(Q_{1}-\left\langle Q_{1}\right\rangle\right)\left(Q_{2}-\left\langle Q_{2}\right\rangle\right)\right\rangle=\left\langle Q_{1} Q_{2}\right\rangle$ of the oscillator amplitudes, however, exhibit oscillations induced by the optical pulse:

$$
\left\langle Q_{i}^{2}\right\rangle=\frac{\hbar}{2 \omega_{i}}\left(1+2 n_{i}\right)-\frac{\hbar}{\omega_{i}} \xi^{i i}\left(1+2 n_{i}\right) \sin 2 \omega_{i} t,
$$

$$
\begin{aligned}
\left\langle Q_{1} Q_{2}\right\rangle= & -\frac{\hbar}{2 \sqrt{\omega_{1} \omega_{2}}} \xi^{12}\left(1+n_{1}+n_{2}\right) \sin \left(\omega_{1}+\omega_{2}\right) t \\
& -\frac{\hbar}{2 \sqrt{\omega_{1} \omega_{2}}} \xi^{12}\left(n_{2}-n_{1}\right) \sin \left(\omega_{1}-\omega_{2}\right) t
\end{aligned}
$$

At finite temperature, the oscillators are not in pure number states $\left|n_{i}\right\rangle$ but in a superposition of those with temperature dependent amplitudes $c_{n_{i}}$, where the sum over their absolute squares is unity: $\left|\psi^{-}\right\rangle=\sum_{n_{1}, n_{2}=0}^{\infty} c_{n_{1}} c_{n_{2}}\left|n_{1}, n_{2}\right\rangle$. To first order in the $\xi^{12}$ this leads to Eq. (3) with the occupation numbers $n_{1}$ and $n_{2}$ replaced by the Bose occupation number expectation values $\left\langle n_{1}\right\rangle$ and $\left\langle n_{2}\right\rangle$ of the two oscillators for the temperature dependent covariance dynamics. The variances (2) oscillate at twice the phonon frequency around their unperturbed values. This behavior is associated with squeezed phonon states. At $T=0 \mathrm{~K}$ this is to first order in $\xi^{i i}$ the result obtained by Garret et al. $[4,5]$. Here, we focus on the consequences of Eq. (3). The covariance of the two amplitudes that is zero without perturbation (i.e., no correlation exists) now contains oscillating contributions with the sum and the difference frequency of the two involved oscillators. The sum-frequency part evolves from perturbation contributions arising from the simultaneous creation or annihilation of two phonons with $\omega_{1}$ and $\omega_{2}$. Creation and annihilation of a phonon at the same time are responsible for the difference-frequency part. Since amplitudes of $\left\langle Q_{1} Q_{2}\right\rangle$ at $\omega_{1}+\omega_{2}$ and $\omega_{1}-$ $\omega_{2}$ are proportional to $1+\left\langle n_{1}\right\rangle+\left\langle n_{2}\right\rangle$ and $\left\langle n_{1}\right\rangle-\left\langle n_{2}\right\rangle$, respectively, the difference-frequency contribution vanishes when $T$ approaches $0 \mathrm{~K}$. This reflects the fact that no phonons are present before the pulse which could be destroyed.

In $\mathrm{KTaO}_{3}$ only second-order, i.e., two-phonon, Raman scattering, is allowed while first-order contributions are forbidden [6]. SOR is accomplished by wave vector conserving combinations of phonon modes from the whole Brillouin zone [7]. The considerations made above for a pair of harmonic oscillators can be applied to any combination of phonon modes as long as wave vector conservation is satisfied. Indices 1 and 2 then have to be replaced by $\mathbf{q}, m$ and $-\mathbf{q}, n$, where $\mathbf{q}$ is the phonon wave vector and $m, n$ are band indices. The transient transmission changes of the sample observed in time-resolved optical experiments can be described by the theoretical framework developed by Yan et al. [8]. There the nonlinear polarization associated with the probe pulse is $P_{k}^{\mathrm{NL}}=$ $\sum_{l} \chi_{k l}^{R S} E_{l}^{\prime}$, with $\mathbf{E}^{\prime}$ the linearly polarized probe field, $\chi_{k l}^{R S}=(2 V)^{-1} \sum_{\mathbf{q}, m, n} \alpha_{k l}^{\mathbf{q}, m, n}\left\langle Q_{\mathbf{q}, m} Q_{-\mathbf{q}, n}\right\rangle \quad$ the secondorder susceptibility tensor, and $V$ the scattering volume. The probe pulse of the temporal width $\tau_{L}$ is assumed to be Gaussian with a center frequency $\omega_{L}: E_{i}^{\prime}(u) \propto$ $\exp \left[-\left(u^{2} / 2 \tau_{L}^{2}\right)\right] \cos \left(\omega_{L} u\right)$ where $u=t-\tau$ and $\tau$ is the time delay between the pump and the probe pulse. Close to the laser's central frequency, the normalized detection frequency dependent transmission changes resulting from the pump induced covariance oscillations are given by

$$
\frac{\Delta T}{T_{0}}=\frac{2 \hbar \pi \tau_{L}^{2} \omega_{L}\left(\omega-\omega_{L}\right)}{n c \sigma} \sum_{\mathbf{q}, m, n} \alpha^{i j} \frac{\xi^{\mathbf{q},-\mathbf{q}, m, n}}{\sqrt{\omega_{\mathbf{q}, m} \omega_{-\mathbf{q}, n}}}\left[A_{\mathbf{q}, m, n}^{+} \cos \left(\omega_{\mathbf{q}, m}+\omega_{-\mathbf{q}, n}\right) \tau+A_{\mathbf{q}, m, n}^{-} \cos \left(\omega_{\mathbf{q}, m}-\omega_{-\mathbf{q}, n}\right) \tau\right]
$$

where

$$
\begin{aligned}
A_{\mathbf{q}, m, n}^{+}= & {\left[1+\left\langle n\left(\omega_{\mathbf{q}, m}\right)\right\rangle+\left\langle n\left(\omega_{-\mathbf{q}, n}\right)\right\rangle\right] } \\
& \times e^{-1 / 2\left(\omega_{\mathbf{q}, m}+\omega_{-\mathbf{q}, n}\right)^{2} \tau_{L}^{2}}\left(\omega_{\mathbf{q}, m}+\omega_{-\mathbf{q}, n}\right)
\end{aligned}
$$

and

$$
\begin{aligned}
A_{\mathbf{q}, m, n}^{-}= & {\left[\left\langle n\left(\omega_{\mathbf{q}, m}\right)\right\rangle-\left\langle n\left(\omega_{-\mathbf{q}, n}\right)\right\rangle\right] } \\
& \times e^{-1 / 2\left(\omega_{\mathbf{q}, m}-\omega_{-\mathbf{q}, n}\right)^{2} \tau_{L}^{2}}\left(\omega_{\mathbf{q}, m}-\omega_{-\mathbf{q}, n}\right)
\end{aligned}
$$

are the amplitudes for the sum- and difference-frequency part and $\sigma$ is the scattering cross section. The total signal therefore is a superposition of a continuum of phononmode combinations from the whole Brillouin zone. The cosinelike signal will be dominated by mode combinations that have a maximum in the combined density of states $N_{\mathbf{q}, m} N_{-\mathbf{q}, n}$. Coherent scattering of the probe pulse redshifts and blueshifts the transmitted spectrum depending

on whether it is in-phase or out-of-phase with an oscillatory contribution to (4) [8]. This results in a linear relation between the signal amplitude and the detection frequency close to $\omega_{L}$ with a sign reversal at $\omega_{L}$.

Experiments are performed on a (100)-oriented $\mathrm{KTaO}_{3}$ $1 \mathrm{~mm}$ thick sample in a standard pump-probe experiment using an ultracompact Ti:sapphire femtosecond laser (GigaJet, GigaOptics, Germany) as pulse source. The $25 \mathrm{fs}$ long pulses are centered at $800 \mathrm{~nm}$ wavelength and delivered at a repetition frequency of $2 \mathrm{GHz}$. An intense pump pulse is focused to a spot of $25 \mu \mathrm{m}$ onto the sample. A second weaker pulse is focused to the same spot. Pumpinduced transmission changes are probed as a function of time delay between the pulses. Average pump and probe powers are 200 and $40 \mathrm{~mW}$, respectively. The probe spectrum is filtered with a monochromator with a bandwidth of $1 \mathrm{~nm}$ behind the sample. Transient transmission changes as a function of detection wavelength are recorded using a 
commercial data acquisition system based on a rapid scanning delay line (Aixscan, GWU Lasertechnik, Germany). The sample is placed in a cryostate.

Figure 1 shows the Fourier transform (FT) intensity spectrum of the time-resolved data at room temperature recorded at a fixed detection wavelength of $835 \mathrm{~nm}$. The time-domain data recorded at a fixed detection wavelength of $835 \mathrm{~nm}$ are depicted in the inset. The signals do not depend on pump or probe beam polarization. The data are dominated by an oscillation with $3.39 \mathrm{THz}$ which matches the overtone of the TA phonon at the zone boundary. It is associated with the generation of squeezed phonon states as shown by Garret et al. [4,5,9]. All higher frequency peaks are identified as covariance oscillations of phonon amplitudes by comparison to the phonon dispersion curves [10] and the continuous-wave Raman spectra of $\mathrm{KTaO}_{3}$ [6]. The peaks at 4.92 and $7.33 \mathrm{THz}$ are associated with $\mathrm{TO}_{1,2}-\mathrm{TA}$ and $\mathrm{TO}_{1,2}+\mathrm{TA}$ mode combinations, respectively, while the peak at $13.24 \mathrm{THz}$ $(16.7 \mathrm{THz})$ corresponds to the $\mathrm{TO}_{4}-\mathrm{TA}\left(\mathrm{TO}_{4}+\mathrm{TA}\right)$ combination mode. The $\mathrm{TO}_{2}$ branch dominates the $\mathrm{TO}_{1,2}$ contributions because of its flatter dispersion associated with a high density of states along a large area of the $\Delta$ direction [10]. TA modes with lower $\mathbf{q}$ values and lower frequencies from the entire $\Delta$ direction are involved in the observed combinations therefore. This explains the rather broad peaks. The frequency difference between these peaks, which should be equal to twice the TA frequency of $3.39 \mathrm{THz}$ for zone-boundary phonons, is at a value of $2.41 \mathrm{THz}$ remarkably reduced, also indicating the contributions from lower $\mathbf{q}$ values. The $\mathrm{TO}_{4}$ branch has a rather steep dispersion compared to the $\mathrm{TO}_{2}$, therefore again zone-boundary mode combinations dominate the signal. This is supported by the fact that the difference of

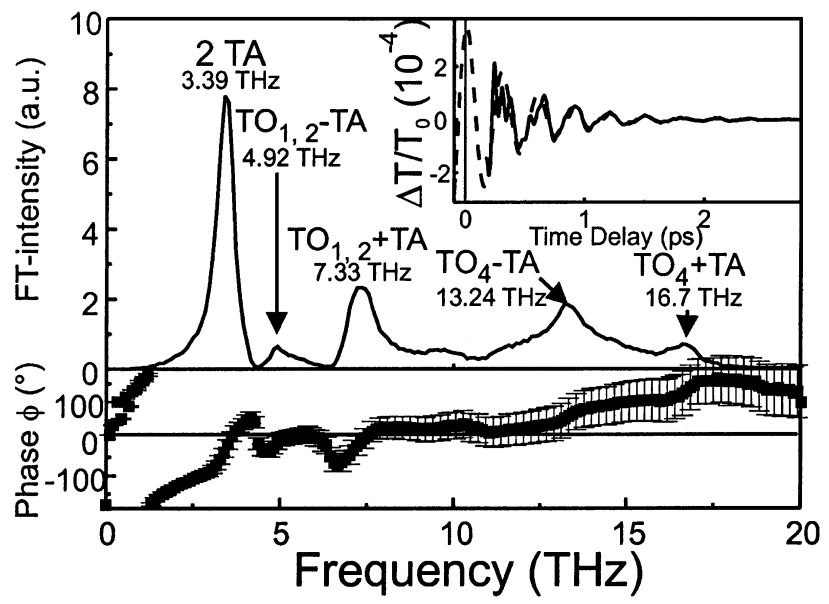

FIG. 1. Fourier intensity and phase spectrum of the transient data at room temperature and a detection wavelength of $835 \mathrm{~nm}$. The excited phonon combinations are indicated in the figure. The phase error is indicated with the error bars. The inset shows the corresponding time-domain data (solid line) and a fit (dashed line) with the 2 TA frequency.
$3.46 \mathrm{THz}$ between the two peaks is almost equal to the zone-boundary $2 \mathrm{TA}$ overtone. The temperature dependence of the amplitudes and frequencies is also taken into account for an unambiguous identification (see below). The phase behavior of the modes will be discussed below.

Figure 2a shows the FT spectra at $835 \mathrm{~nm}$ detection wavelength for temperatures between 290 and $10 \mathrm{~K}$. The shifts of the phonon frequency with temperature are the same as determined by Nilsen et al. [6]. The amplitudes of selected phonon combinations are also plotted versus temperature in Fig. 2b. Obviously some peaks vanish at low temperatures while others survive. The peaks that vanish for $T \rightarrow 0$ correspond to the difference-frequency combinations $\mathrm{TO}_{1,2}-\mathrm{TA}$ and $\mathrm{TO}_{4}-\mathrm{TA}$. The sum-frequency contributions remain well pronounced at low temperatures.

To compare the experimental temperature dependence of the FT amplitudes (square roots of the peak values in the intensity spectra) to the theory, they are corrected for the oscillation frequency dependent contribution to the $A_{\mathbf{q}, m, n}^{ \pm}$in the detection term (4) and normalized to unity
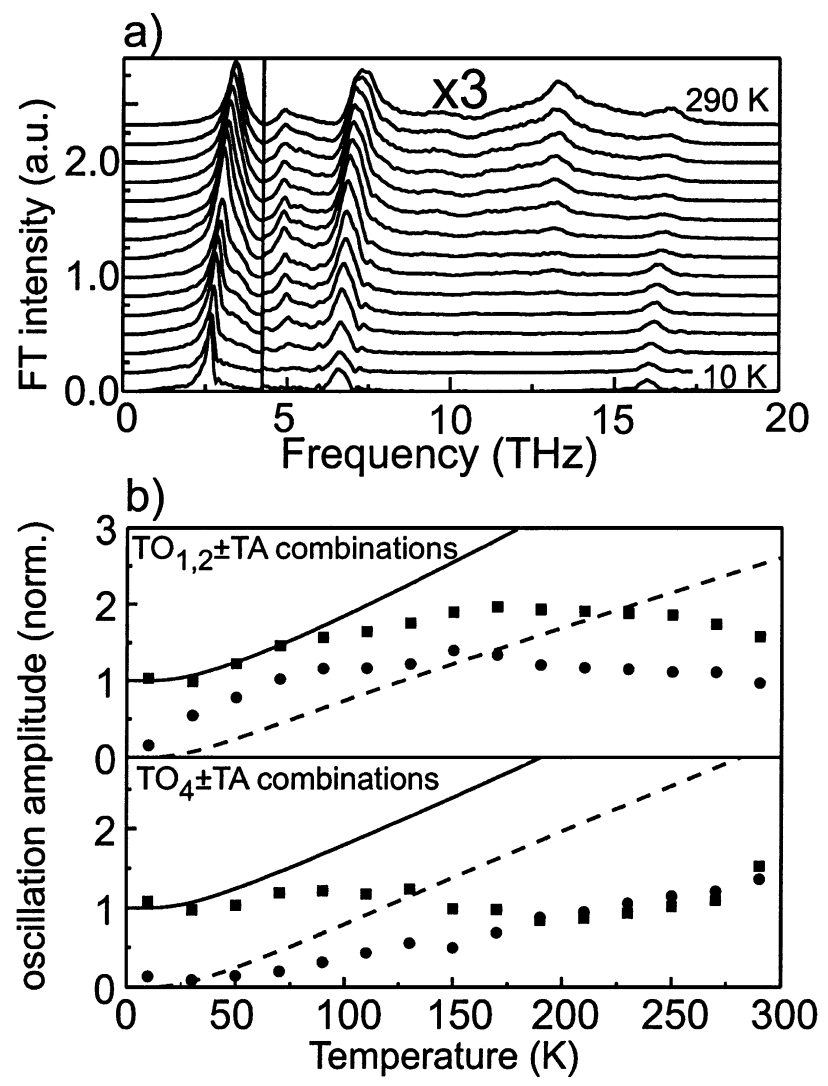

FIG. 2. (a) Fourier intensity spectra of the transient transmission changes at temperatures between 290 and $10 \mathrm{~K}$. The part of the spectra on the right-hand side of the vertical line has been enlarged by a factor of 3. (b) Normalized and corrected (see text) temperature dependent amplitudes of the $\mathrm{TO}_{1,2} \pm \mathrm{TA}$ and $\mathrm{TO}_{4} \pm \mathrm{TA}$ combinations. Squares (circles) correspond to sum frequencies (difference frequencies). Solid and dashed lines are calculated amplitudes of the sum-frequency (solid line) and difference-frequency (dashed line) contribution. 
for the sum-frequency amplitude at $T \rightarrow 0$. These values are depicted as a function of temperature in Fig. $2 b$ in comparison to the theoretical values, $1+\left\langle n_{1}\right\rangle+\left\langle n_{2}\right\rangle$ and $\left|\left\langle n_{1}\right\rangle-\left\langle n_{2}\right\rangle\right|$. The basic features are reproduced by the simple model; i.e., the difference-frequency amplitude approaches zero for $T \rightarrow 0$ and both difference and sum contributions increase for higher temperatures. The agreement is qualitatively good for $T<50 \mathrm{~K}$. For higher temperatures the data deviate significantly from the model. We attribute this in part to phonon-phonon interaction which leads to an increased decay of the covariance amplitudes at elevated temperatures as observed in the broadening of the peaks in Fig. 2. However, some details of the temperature dependence of the amplitudes are presently not understood, e.g., the drop and subsequent rise of the $\mathrm{TO}_{4}+\mathrm{TA}$ amplitude above $200 \mathrm{~K}$.

In addition to the amplitudes, we determine the phases of the variance and covariance oscillations. A fit with a single damped harmonic oscillation with the 2 TA frequency to the room temperature signal is shown in the inset of Fig. 1. A cosinelike behavior as predicted by Eq. (4) for the variance oscillation is observed. The phase plot in Fig. 1 shows that indeed all contributions to the signal below $\approx 14 \mathrm{THz}$ are cosinelike $(\phi=0)$ in agreement with the theory. For higher frequencies the phase error becomes too large for an unambiguous determination of the phases since the uncertainty in the determination of zero time delay is approximately $10 \mathrm{fs}$. Equal phase behavior is observed for all temperatures.

An important confirmation for the proposed detection process of the oscillations is the $\omega$ dependence of the amplitudes according to Eq. (4). The inset of Fig. 3 shows the amplitude of the $\mathrm{TO}_{1,2}+\mathrm{TA}$ contribution as a function of the detection wavelength together with the pulsed optical spectrum. A linear behavior in the vicinity of the laser's central frequency with a $\pi$ phase shift at the central frequency is observed for this and all other modes [9]. The sign reversal of all features of the signal is impressively demonstrated in Fig. 3 for detection wavelengths of 749 and $850 \mathrm{~nm}(T=10 \mathrm{~K})$. These results demonstrate that the detection in the spectrally resolved transmission scheme occurs via a transient redshift and blueshift of the pulsed spectrum. This result is confirmed in a spectrally integrated measurement detecting the whole probe spectrum. In this case all contributions cancel out and no oscillations are detectable although the resolution limit of the setup is $10^{-9}\left(\Delta T / T_{0}\right)[11]$.

In conclusion, we have driven combination states of phonon pairs out of the whole Brillouin zone of $\mathrm{KTaO}_{3}$ via SOR by excitation with a fs laser pulse. Oscillators that are decoupled in the absence of the impulsive perturbation become correlated resulting in oscillations of the amplitude covariance. Their temperature dependence, i.e., vanishing difference frequency combinations and increasing ampli-

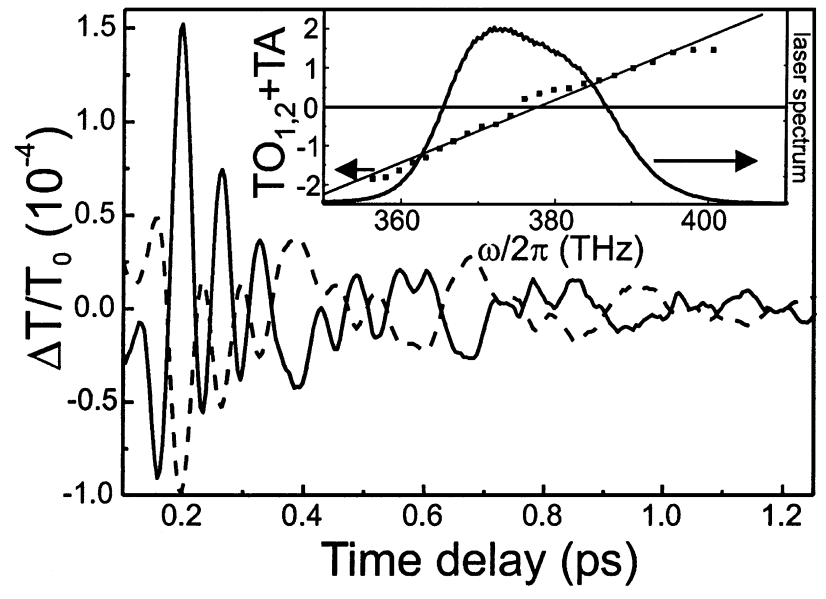

FIG. 3. Transient transmission changes of the sample for detection wavelengths of $850 \mathrm{~nm}$ (solid line) and $749 \mathrm{~nm}$ (dashed line). The inset shows the wavelength dependent amplitude of the $\mathrm{TO}_{1,2}+\mathrm{TA}$ oscillation and the laser spectrum. The straight solid line is a guide to the eye.

tudes with increasing temperature, can be explained by a quantum mechanical model.

This work is supported by the Deutsche Forschungsgemeinschaft. We thank A. Stahl, M. Artoni, and W. Xu for helpful discussions. A. B. thanks the Konrad-Adenauer Stiftung for support.

*Email address: bartels2@iht-ii.rwth-aachen.de

[1] G. C. Cho et al., Phys. Rev. Lett. 65, 764 (1990); T. K. Cheng et al., Appl. Phys. Lett. 57, 1004 (1990).

[2] For a review see T. Dekorsy et al., in Light Scattering in Solids VIII, edited by M. Cardona and G. Güntherodt (Springer, Berlin, 2000), and references therein.

[3] X. Hu and F. Nori, Phys. Rev. B 53, 2419 (1996); X. Hu and F. Nori, Phys. Rev. Lett. 79, 4605 (1997); X. Hu and F. Nori, Physica (Amsterdam) 263-264B, 16 (1999); see also M. Artoni, J. Nonlinear Opt. Phys. Mater. 7, 241 (1998).

[4] G. A. Garret et al., Science 275, 1638 (1997).

[5] G. A. Garret et al., Opt. Exp. 1, 385 (1998).

[6] W. G. Nilsen and J. G. Skinner, J. Chem. Phys. 47, 1413 (1967).

[7] R. Loudon, Phys. Rev. 137, A1784 (1965).

[8] Y.X. Yan et al., J. Chem. Phys. 83, 5391 (1985).

[9] Our results show that the variance signal drastically depends on the detection wavelength as expected from Eq. (4). Reference [4] uses a term of second order in $\omega$ in the description of the detection process that is the same as the one we used in the relevant wavelength range. In the experimental part of Ref. [4], however, no reference to a detection wavelength is given, though the amplitude of the oscillations and hence the determined squeezing amplitude should strongly depend on it.

[10] C. H. Perry et al., Phys. Rev. B 39, 8666 (1989).

[11] A. Bartels et al., Opt. Lett. 24, 996 (1999). 